\title{
Regard
}

\section{Origine et évolution des langues. Débat sur la construction des connaissances à travers l'analyse d'un ouvrage}

\author{
Robert Nicolaï \\ Linguiste, membre de l'Institut universitaire de France, Université de Nice Sophia Antipolis, UFR LASH, \\ 98 boulevard Édouard-Herriot, BP 3209, 06204 Nice cedex 3, France
}

\begin{abstract}
Périodiquement, NSS offre à ses lecteurs un éclairage sur les recherches interdisciplinaires en cours dans des domaines pouvant apparaître marginaux par rapport au centrage habituel de la revue, mais qui n'en sont pas moins essentiels pour comprendre l'évolution de notre espèce et la place qu'elle occupe sur notre planète.

Ce texte écrit par un linguiste développe, à partir de l'analyse d'un ouvrage récent, les enjeux socioscientifiques et épistémologiques que provoque la participation de différentes disciplines (linguistique, génétique, anthropologie, archéologie, sciences cognitives) dans les recherches sur l'origine et l'évolution des langues.

Il s'agit ici de jauger des hypothèses qui ont connu un succès médiatique certain. Dans les années 1990, la controverse a d'abord porté sur une reconstruction fortement contestée d'une langue originelle unique. À la même époque, les sciences cognitives s'affirmaient. L'hypothèse intitulée "Nouvelle Synthèse ", fondée sur l'affirmation d'une corrélation entre la transmission des gènes et celle des langues, a été appliquée, en particulier, à la diffusion de l'agriculture en même temps qu'elle a tenté de rendre compte de la division des langues indo-européennes.

Cependant, comme nous le montre l'auteur à travers son analyse, la réalité est plus complexe parce qu'il y eut souvent disjonction entre la transmission des gènes, qui est biologique, et celle de la langue, qui est culturelle, et parce que, tout particulièrement, les modalités utilisées pour l'analyse des données linguistiques ne souscrivent pas aux exigences de l'objectivité scientifique. La difficulté est de reconstituer le passé en s'appuyant sur des disciplines multiples, à partir de faits observables aujourd'hui et de simples conjectures pour les temps anciens ; elle est aussi dans l'évaluation des contraintes socioscientifiques qui surdéterminent ce type de recherche, ce qui donne toute son importance à la réflexion épistémologique sur les transformations de nos cadres disciplinaires et de nos pratiques de recherche.
\end{abstract}

La Rédaction

\section{L’ouvrage}

Lorsqu'on aborde un ouvrage sur la thématique de l'origine du langage et de l'évolution des langues, qui a ressurgi avec force dans les dernières décennies du XXe siècle, c'est avec curiosité mais aussi avec l'appréhension de se trouver face à l'exposé irréfléchi, dogmatique et convenu de vulgates aussi peu scientifiquement fondées que médiatiquement propulsées à grand renfort de moyens et d'articles vulgarisateurs supposés ouvrir - sinon « libérer » - la recherche dans le domaine. Origin and Evolution of Languages. Approches, Models, Paradigms, édité par Bernard Laks avec Serge Cleuziou, Jean-Paul Demoule et Pierre Encrevé (London, Equinox, 2008) n'a rien de tout cela et propose au contraire un large panorama de la question ; c'est pourquoi je m'attacherai à son

Auteur correspondant : nicolai@unice.fr analyse avant d'orienter le débat vers les problèmes qu'il contribue à soulever.

Comme ses éditeurs le mentionnent, l'ouvrage rassemble une partie des communications présentées en 2002 lors d'un colloque au Collège de France. En ambitionnant de réunir des spécialistes, internationalement connus, de linguistique, d'anthropologie, d'archéologie, de génétique et de la cognition, l'objectif était de « confronter [...] les différentes hypothèses et les différents modèles proposés pour rendre compte de l'origine et de l'évolution des langues", en souhaitant que les débats ainsi ouverts permettent « de rendre compte de la diversité des positions défendues, tout en montrant l'existence de nombreuses convergences disciplinaires et interdisciplinaires ». Cela étant, la moitié seulement des communications présentées ont donné lieu à un chapitre et, a posteriori, les éditeurs ont décidé d'intégrer 
au volume plusieurs compléments (chapitres de Lyle Campbell, S. Cleuziou, Bernard Comrie, J.-P. Demoule, B. Laks, Frederick J. Newmeyer) pour équilibrer l'ensemble et pallier une sous-représentation des points de vue qui apportent quelque distance face aux exposés de ces "nouvelles avancées »; les contributions ainsi ajoutées par les éditeurs (dont les leurs) introduisent une dimension critique face aux thèses standard des tenants de la « Nouvelle Synthèse ». Le volume est organisé en deux sections (intitulées «ab originem » et «post originem») selon que l'accent est mis sur l'origine du langage ou sur le développement des langues; ce ne sera cependant pas cette division-là que je suivrai. Rompant la succession imposée des chapitres, j'adopterai d'autres regroupements pour faire ressortir les problématiques et les cadres conceptuels sous-jacents.

\section{« Nouvelle Synthèse »1}

L'annonce du colloque, dont cet ouvrage est issu, commençait ainsi :

«Les recherches sur les origines de l'homme ont été spectaculairement relancées depuis une dizaine d'années par les travaux d'équipes de généticiens, de linguistes, d'anthropologues et d'archéologues, qui ont abouti à la proposition d'un nouveau modèle d'ensemble. [...] Avec leurs méthodes propres, et à partir des langues et des gènes actuels, certains courants de la génétique des populations et de la linguistique typologique sont ainsi parvenus à proposer une reconstitution des arbres généalogiques respectifs des langues et des gènes de l'ensemble de l'humanité, tandis qu'un certain nombre d'archéologues proposaient de corréler ces arbres avec des migrations préhistoriques attestées, notamment lors de la diffusion de l'agriculture au cours du Néolithique. »

Ce qui était proposé était donc un état des lieux du programme dit de la « Nouvelle Synthèse », pris comme moteur d'un renouvellement de la recherche. Passée l'introduction, l'ouvrage commence par un chapitre de L.L. Cavalli-Sforza qui réitère ses hypothèses, largement médiatisées par ailleurs, selon lesquelles les humains modernes seraient les descendants d'un petit groupe localisé en Afrique de l'Est, il y a environ 100000 ans. Par

\footnotetext{
${ }^{1}$ Le nom de « Nouvelle Synthèse » a été donné par Colin Renfrew (archéologue, Université de Cambridge, RoyaumeUni) à la convergence pluridisciplinaire de ses travaux avec ceux de Luigi Luca Cavalli-Sforza et de Merritt Ruhlen (respectivement généticien et linguiste, Université Stanford, ÉtatsUnis). On peut, ainsi que le fait Métoz (2006), la résumer en disant que ces « scientifiques se donnent comme objectif ultime de comprendre l'origine de l'Homme par l'étude de sa diversité génétique, linguistique et culturelle et de prouver la monogenèse des langues du monde et de l'espèce humaine ", avant de décrire les méthodologies employées et de montrer les limites des arguments avancés.
}

vagues successives, ils se seraient ensuite répandus, colonisant l'ensemble de la planète et conduisant probablement à la disparition des autres espèces proches en raison d'avantages sélectifs à leur disposition, dont la faculté de langage est sans doute le plus saillant. Supposant que le langage s'est développé chez les humains avant leur «sortie » d'Afrique, il est dès lors admis qu'il y a une origine unique pour l'ensemble des langues actuellement parlées. Muni de ce préalable, l'auteur synthétise les propositions connues concernant une corrélation entre les arborescences construites par les généticiens et celles élaborées par certains linguistes (Greenberg, 1963 et 1987 ; Ruhlen, 1994) pour rendre compte des familles de langues. Toutefois, il reconnaît un point qui pose problème et qui a créé des exceptions à la corrélation langues-gènes : celui de la transmission culturelle (horizontale) des langues à travers les événements sociaux, économiques, politiques. Ensuite, après avoir inventorié différents exemples de changement de langue et souligné que l'histoire des expansions des humains modernes, qui est fondée sur des données archéologiques et génétiques, peut être utile pour comprendre l'histoire de la diffusion des langues, il aborde le cas de l'expansion de l'agriculture et de la diffusion des langues indo-européennes selon les hypothèses de $\mathrm{C}$. Renfrew (Bellwood et Renfrew, 2002).

Cela étant, les vastes hypothèses de la « Nouvelle Synthèse » sont loin d'être satisfaisantes pour tous. Ainsi, face à la question cruciale de l'existence d'une corrélation entre arbres génétiques et linguistiques, B. Comrie ${ }^{2}$ (chapitre 2) souligne une évidence : "One can readily recognize that there is no logical necessity for languages and genes to correlate. Genes are transmitted biologically [...]. Language [...] is transmitted culturally [...]. ». Et, après avoir noté : "It is an empirical question to what extent such social phenomena have disrupted the close correlation between language classification and population genetic classification in the history of humankind», il illustre son propos en mettant en regard faits linguistiques et faits de génétique des populations à Madagascar, en Nouvelle-Guinée et dans le Caucase, montrant des phénomènes qui mettent à mal l'hypothèse de la corrélation langues-gènes et qui ne s'expliquent que par des emprunts de langues. Dès lors, il retient la nécessité d'engager des recherches empiriques pour cerner les variables concernées et appréhender les circonstances ethnographiques et archéologiques dans lesquelles arbres linguistiques et génétiques sont susceptibles de se correspondre.

Mais c'est aussi à l'intérieur du domaine linguistique que les arborescences proposées par $\mathrm{M}$. Ruhlen à la suite de J. Greenberg ont fait débat. Elles sont non seulement loin de faire l'unanimité des linguistes (ce qui n'est pas grave), mais, surtout, elles sont loin de satisfaire à des

\footnotetext{
${ }^{2}$ Linguiste, Institut Max-Planck d'anthropologie évolutionniste, Allemagne.
} 
critères de scientificité suffisants pour être acceptées sans réserve (ce qui est plus grave). La mise en parallèle du texte de M. Ruhlen (chapitre 10), qui présente, une nouvelle fois, les hypothèses fortement médiatisées qu'il a précédemment développées (1994), et de la critique que lui oppose L. Campbell ${ }^{3}$ (chapitre 4) illustre ce débat. Il ne sera pas utile de reprendre le détail des hypothèses de M. Ruhlen. Rappelons simplement qu'il s'emploie à contester les positions comparatives classiques, qui posent qu'il n'est pas possible pour des raisons pratiques de remonter au-delà de la barrière de 5000 ans dans la reconstruction des langues (date des premiers documents écrits), et qu'il reprend le schéma de J. Greenberg, qui, après avoir initié sa méthodologie sur les langues africaines (1963), a attendu que les controverses soient calmées pour poursuivre avec sa macrofamille eurasiatique et ses trois familles amérindiennes (1987). C'est à cette approche-là que s'oppose frontalement L. Campbell. En réponse à la question : "Que pouvons-nous découvrir à propos de l'état le plus ancien du langage en partant de la comparaison des langues actuelles? » il répond que l'on ne peut rien découvrir parce que trop de changements ont eu lieu pendant trop longtemps. Rien de la (ou des) langue(s) originelle(s) ne survit dans les langues modernes sous des formes dont la comparaison pourrait donner des indications utiles sur le contenu lexical ou structural de la (ou des) langue(s) originelles(s). De fait, cette controverse a fait l'objet de grands débats dans les années $1990^{4}$, et ce qui est présenté ici n'en est que la reprise pour un nouveau public.

Enfin, dans le domaine archéologique, J.-P. Demoule ${ }^{5}$ (chapitre 9) analyse l'hypothèse tout autant médiatisée de C. Renfrew sur 1'origine des Indo-Européens et leurs migrations en rapport avec la diffusion de l'agriculture. Dans la lignée des critiques qu'il a très tôt développées par ailleurs (1980), l'auteur discute une série de points pour lesquels il ne trouve pas de réponse satisfaisante chez C. Renfrew et il conclut : «[...] his hypothesis is not verifiable-or it is not 'falsifiable' [...] i.e. there exists no means of proving that is possibly false. In the best of cases, one could say 'why not?' ; but equally well, 'why?'. » Il note cependant une évolution de l'approche de Renfrew (1999), lorsque celui-ci n'exclut plus l'emprunt et adopte une certaine dose de convergence au sein de son modèle arborescent, en empruntant la notion de «Sprachbund 》 (Troubetzkoy, 1939), qui lui sert dès lors à répondre à certaines critiques telles que le fait que le modèle linéaire de la diffusion de la colonisation néolithique ne correspond à aucun des arbres linguistiques élaborés par les linguistes.

Pour clôturer ce voyage en «Nouvelle Synthèse » et en s'appuyant sur l'espace mésopotamien, où l'on situe, il y

\footnotetext{
${ }^{3}$ Linguiste, Université d'Utah, États-Unis.

${ }^{4} \mathrm{Cf}$. les discussions sur le forum bien connu des linguistes : http://linguistlist.org.

5 Archéologue, Université Panthéon-Sorbonne, Paris.
}

a environ 5000 ans, la naissance de l'écriture et donc la possibilité de l'histoire, S. Cleuziou ${ }^{6}$ (chapitre 13) apporte le point de vue de l'archéologue en posant la question essentielle : "How far back can we go from known cultural assemblages to languages, and how can we do it? » Et il conclut qu'appréhender les langues à travers l'archéologie est une tâche impossible, car on ne saurait passer simplement de la saisie de cultures matérielles aux langues, ce qui peut être rendu dans les termes plus modérés d'un questionnement : Existe-t-il des moyens préférentiels pour passer des cultures aux langues, ou bien cela reste-t-il un insurmontable défi?

\section{Approche cognitive}

Nous avons fait là le tour des points cardinaux de la « Nouvelle Synthèse », mais cela n'épuise pas le questionnement sur l'origine du langage, qui croise ceux ouverts par les recherches cognitives. Certes, la relation n'est pas directe et il s'agit sans doute d'une rencontre conjoncturelle. Toutefois, une réflexion sur l'émergence du langage, conduite dans une visée plus ou moins néodarwinienne, introduit des connexités suffisantes pour que se pencher sur cette question puisse paraître légitime à des linguistes qui conjecturent sur le développement cognitif en rapport avec le langage. Les questions posées portent alors sur l'émergence et la place de cette faculté de langage et sur sa complexification, sur les conditions de l'évolution des langues, sur les modalités de constitution de la grammaire et de l'acquisition des propriétés syntaxiques complexes, sur l'existence première ou non d'une structure conceptuelle, sur l'importance des représentations conceptuelles par rapport au procès de communication et aux pressions communicatives corrélativement manifestées, etc. Trois chapitres touchent à ces questions.

Ainsi, Andrew Carstairs-McCarthy ${ }^{7}$ (chapitre 3) constate qu'en raison de notre non-extériorité par rapport au langage, nous ne pouvons pas savoir si tel aspect ou telle configuration linguistique particulière, bien qu'universellement attestée, est générale au sens où nous devons nous attendre à la trouver quelle que soit l'espèce qui utiliserait le langage, ou bien si c'est un accident de l'histoire au sein de l'espèce, un "poor designed feature » (une caractéristique qui ne correspond à aucun avantage évolutif et qui a perduré parce que rien ne s'y est opposé, tel le croisement des systèmes respiratoire et digestif des mammifères). Il se réfère alors au biologiste Williams (1992), dont il reprend la classification des principes d'explication de l'évolution des organismes : (1) pression adaptative; (2) principes généraux; (3) accidents de l'histoire. Proposant ensuite de penser

\footnotetext{
6 Archéologue, Université Panthéon-Sorbonne, Paris.

7 Linguiste, Université de Canterbury, Nouvelle-Zélande.
} 
l'évolution du langage en des termes parallèles, il la rapporte à la division en trois classes proposée par Chomsky (2001), qui, à propos des conditions initiales de l'acquisition du langage, distingue : (i) les éléments inexpliqués de l'état initial génétiquement déterminé de la faculté de langage ; (ii) les conditions et les principes qui concernent les liens entre les expressions de langue et les systèmes sensorimoteurs et cognitifs ; (iii) les propriétés générales physicochimiques et formelles. Mais, contrairement au point de vue chomskien, selon lequel rien d'intéressant ne peut être dit sur les aspects du langage qui correspondent à la catégorie des « accidents de l'histoire »- (3) chez Williams, (i) chez Chomsky -, l'auteur postule que c'est cette catégorie-là qui est la plus révélatrice de la façon dont le langage a émergé. Il met l'accent sur son importance à travers la discussion de quelques exemples appropriés, qu'il aborde selon la modalité d'une expérience de pensée, en vue d'évaluer leur valeur plus ou moins avantageuse pour la communication linguistique.

Avec la contribution de Frederick J. Newmeyer ${ }^{8}$ (chapitre 5), nous nous situons sur un autre plan. Considérant qu'il existe deux hypothèses concernant le développement de la grammaire, qui serait fondé soit sur une structure conceptuelle, instrument de la pensée ("position classique»), soit sur la capacité à véhiculer la communication humaine, l'auteur défend la «position classique », mais propose la succession suivante: «First, there existed a level of conceptual structure [...]. Secondly came the principal evolutionary event. Conceptual structure was linked to the vocal output channel, creating for the first time a grammar that was independent of the combinatorial possibilities of conceptual structure per se, and making possible the conveying of thought. That is, it made vocal communication possible. »

Confrontés à leur tour à la question « D'où vient le langage? » Gilles Fauconnier ${ }^{9}$ et Mark Turner ${ }^{10}$ remarquent que cela revient à poser la question de l'origine des capacités cognitives de l'esprit humain moderne. Dès lors, ils en reviennent à leur modèle "conceptual integration theory » (Fauconnier et Turner, 2002), qui est censé montrer que les humains cognitivement modernes sont pourvus d'une forme avancée d'opération mentale ( double-scope integration») qui leur permet de développer de nombreuses singularités : art, musique, science, modes d'habillement, danse, mathématiques. Les auteurs postulent que les singularités humaines se développent comme des produits de cette "double-scope integration » et ils vont s'intéresser aux implications de ces résultats (hypothèses) à propos de l'origine du langage. Pour eux, les humains deviennent cognitivement modernes lorsqu'ils acquièrent cette capacité et le langage

\footnotetext{
${ }^{8}$ Linguiste, Université de Washington, États-Unis.

9 Linguiste, Université de Californie à San Diego, États-Unis.

10 Professeur en sciences cognitives, Université Case Western Reserve, États-Unis.
}

humain en est un produit. Les auteurs postulent donc que l'évolution a fourni une capacité qui, une fois qu'elle a atteint le stade de la «double-scope integration », possède la grammaire comme produit.

Alors que ces chapitres développent des conjectures et des modèles à propos des procès cognitifs censés avoir conduit au développement de la faculté de langage, puis à l'émergence des capacités grammaticales entraînant le développement des langues, Salikoko S. Mufwene ${ }^{11}$ (chapitre 12) se situe aux marges et pose la question : "What do creoles and pidgins tell us about the evolution of language? » Pour lui, les développements des créoles et des pidgins n'ont rien qui puisse permettre de les rapprocher des conditions qui ont conduit à l'émergence préhistorique des langues modernes.

\section{Modélisation}

Arrivé à ce stade, il reste à s'intéresser à la dernière thématique de l'ouvrage : la modélisation. Deux chapitres y sont consacrés.

Dans le premier, Domenico Parisi, Francesco Antinucci, Francesco Natale et Federico Cecconi ${ }^{12}$ (chapitre 8) proposent une modélisation de l'approche de C. Renfrew. Ils définissent leur principal objectif comme méthodologique et présentent une simulation qui a pour fonction d'essayer de reproduire (expliquer [sic]) l'expansion de l'Empire néoassyrien dans l'ancien Proche-Orient et le procès de peuplement de l'Europe selon l'hypothèse de C. Renfrew.

Dans le second, Don Ringe ${ }^{13}$ et Tandy Warnow ${ }^{14}$ (chapitre 11) présentent une intéressante approche dans le cadre d'une modélisation et d'une terminologie cladistiques. Il s'agit pour eux de dépasser les modèles arborescents classiques et d'intégrer les phénomènes du contact qui ont été fortement sous-estimés. Ils développent ainsi des propositions, qu'ils mettent à l'épreuve d'un certain nombre de situations de contact, triviales ou non, telles celles attestées dans des langues comme le michiff ou le mednyj-aléout.

\section{Distance}

Il reste enfin le chapitre de B. Laks ${ }^{15}$ (chapitre 7), version anglaise d'un précédent article (2002) qui propose

\footnotetext{
11 Linguiste, Université de Chicago, États-Unis.

12 Institut des sciences et des technologies de la cognition, Italie.

13 Professeur de linguistique, Université de Pennsylvanie, États-Unis.

14 Professeur au département des sciences informatiques, Université du Texas, États-Unis.

15 Professeur de sciences du langage, Université Paris Ouest Nanterre La Défense, France.
} 
une bonne réflexion sur les outils et les méthodes utilisés pour la recherche sur l'origine et l'évolution des langues. L'auteur prend une certaine distance historique et épistémologique. Après avoir commenté le modèle généalogique et la représentation en arbre, «qui ne demandent qu'à réapparaître » car, "plus que des hypothèses attachées à un cadre théorique particulier, ils constituent une construction doxique prompte à faire surface dès que l'interrogation sur l'origine se trouve réactivée ", il procède à une présentation du modèle comparatiste classique et de ses alternatives, puis revient sur l'importance des mélanges de langues et critique la perspective nominaliste (definitio rei). Parallèlement, il réfléchit sur certaines modalités sociologiques du «come-back» du comparatisme généalogique, telles que la référence récurrente et souvent agressive à l'article 2 de la Société de linguistique de Paris - qui précisait en 1866 que la Société n'admettrait aucune communication concernant l'origine du langage -, notant que l'argument " situe le débat sur le terrain de la lutte de la pensée scientifique libre contre la sclérose du politiquement correct institutionnalisé par l'Université »; il souligne aussi les outrances et les carences de certains zélateurs, dont M. Ruhlen. Sans doute l'auteur n'aura-t-il pas utilisé toutes les références dont il pouvait disposer lorsqu'il a développé sa critique (Nicolaï, 2000 et 2006), mais cela n'enlève rien à la pertinence de son propos. Après un exposé bien documenté, il conclut par une "ouverture», précisant que «la métaphore du Stammbaum et la vulgate grossière du darwinisme sur lequel elle se fonde sont loin de constituer les cadres nécessaires de toute réflexion sur l'histoire et l'évolution des langues » et que «c'est bien par la déconstruction critique d'outils qui trop longtemps ont encombré le terrain historique ou typologique que passe aujourd'hui le renouveau de ces recherches ». Ce qui nous entraîne à réfléchir à ces questions et à analyser les vecteurs de leur diffusion, dont ce livre est un exemple manifeste.

\section{Le débat}

Tous les chapitres de cet ouvrage sont écrits par des personnalités de renom international. Toutefois, et c'est sans doute l'une des qualités des éditeurs que de s'être attachés à le montrer, il résulte de sa lecture que, si les thèmes abordés ont " secoué » une partie du milieu scientifique, c'est davantage par la façon de poser les questions (sinon de les imposer) que par les réponses obtenues. Il y a là un paradoxe qui fait que ce livre, donné pour présenter des résultats (y compris dans leurs contradictions), constitue une trace "symptomatique » de questionnements et de pratiques de recherche plutôt qu'il ne rend compte de connaissances empiriques. En effet, il ne présente pas des résultats de "science froide», comme on l'attendrait d'un manuel - et c'est heureux : ce n'est pas un manuel. Il ne présente pas pour autant un état de "science chaude», car celle-ci ne se présente pas : elle se fait. Malgré la saine distance affichée par les éditeurs - et peut-être en raison de cet affichage même -, cet ouvrage est un outil, actif dans le procès général de transformation dont il ambitionne de montrer l'importance et l'intérêt. En conséquence, plusieurs questions se posent, d'ordre sociologique, scientifique et épistémologique.

\section{Opportunité sociologique}

Ainsi, on pourra demander : À qui les auteurs s'adressent-ils? À quelle demande répondentils? Qu'est-ce qu'illustre le contenu de l'ouvrage? Qu'apporte-t-il à la communauté des lecteurs? Et il n'y a aucune réponse simple à ces questions.

Il se donne comme un état de l'art, une étape de la réflexion du moment. Mais à l'usage de qui ? Certainement pas des auteurs, qui, dans leur grande majorité, n'ont fait que reprendre ou reformuler des textes dont le contenu date d'un quart de siècle pour les plus emblématiques d'entre eux, et qui sont donc parfaitement connus de leurs pairs. Nous n'avons pas affaire pour autant à un ouvrage qui viserait l'exhaustion d'une problématique afin de la rendre assimilable par un public étudiant : il y manquerait la visée pédagogique. Nous n'avons pas non plus affaire à un livre de vulgarisation pour le grand public.

N'étant rien de tout cela, cet ouvrage est néanmoins un peu tout cela. Dès lors, il est intéressant de tenter de le situer par rapport aux contraintes socioscientifiques de la recherche actuelle. Il ressemble à un outil forgé à l'intention d'un public académique, soit donc à un ouvrage a priori conçu à l'intention des pairs de ses auteurs-éditeurs et des instances évaluatrices de l'activité de production et de «rayonnement» scientifiques conduite dans le hic et nunc. Et l'on sait que la reconnaissance et la sanction de cette activité de production sont aujourd'hui une nécessité pour le développement de la recherche individuelle et collective, pour la poursuite et la conduite des projets scientifiques souhaités et pour le développement de l'image et de la carrière de ses acteurs. Sans doute, l'une des dimensions de l'ouvrage est donc déterminée par la nécessité de se situer dans les courants de recherche d'aujourd'hui, de positionner une problématique et de se présenter comme existant par rapport à elle - ce qui est normal. Pratiquement, cela ne peut pas ne pas passer par l'occupation d'un terrain et une présentation de soi manifestée par des postures et la circulation de publications scientifiques qui attestent (de) cette existence. L'ouvrage a donc une fonction de cristallisation : il est à la fois une pierre blanche qui marque le cheminement séculier des chercheurs qui l'ont produit, une balise qui matérialise un état des propositions et des points de vue du moment et, de par l'origine de sa profération et les qualités de ses 
auteurs, le symbole d'un "moment d'excellence » dans un océan médiatique que la recherche scientifique subit, génère, utilise à la fois, et appelle le cas échéant.

\section{Validité scientifique}

Sur ce plan, les questions pourraient être : Quelle est la valeur et 1'originalité de ces présentations? Quelles ouvertures autorisent-elles? Quel type de critique peuton faire à leur propos?

Se déterminer sur la valeur scientifique d'un tel ouvrage conduit à une analyse paradoxale, car ce n'est qu'à l'intérieur de chacun des domaines disciplinaires parties prenantes du programme (linguistique comparative, cognition, typologie des langues, génétique des populations, archéologie, modélisation informatique) que l'évaluation scientifique est légitime. Et elle se fait par la critique des pairs. L'ouvrage montre bien, à travers ses exemples emblématiques (Comrie / Cavalli-Sforza, Campbell / Ruhlen, Demoule/Renfrew), que cette critique peut être précise et riche d'enseignements. Or, la problématique de la «Nouvelle Synthèse » se fonde sur un programme interdisciplinaire et, au-delà, sans doute sur l'ambition de contribuer à une recomposition des domaines de recherche. Dont acte. C'est alors dans un espace volontairement reconstruit, avec de nouveaux « allant de soi » et de nouveaux «étant donné » pris pour base de construction de connaissances futures, que l'on est conduit à se mouvoir. Mais ce n'est pas cela qui fait problème, car la plupart des «avancées de la connaissance » reposent sur des procès de déconstruction-reconstruction impliquant mises en question, reconceptualisations et, à terme, une transformation des connaissances, une mise en perspective des nouveaux résultats avec, le cas échéant, une analyse de leur compatibilité théorique et/ou empirique. Or, ici, ce qui est intéressant, c'est que ce n'est pas sur des acquis empiriques que le procès de déconstruction-reconstruction se fonde; quel que soit le domaine abordé (langage, archéologie, cognition), c'est sur de simples conjectures et sur des hypothèses non réfutables que les nouvelles propositions scientifiques vont s'appuyer.

Certes, il est évident qu'une dose de non-réfutabilité est toujours présente à la base des "paradigmes » que nous élaborons pour appréhender le monde, le comprendre et en rendre compte (saurait-on envisager une réfutabilité des hypothèses darwiniennes?). Mais il importe de distinguer entre une non-réfutabilité originelle, à rapprocher d'une hypothèse de départ ou d'une position axiomatique, et une non-réfutabilité subséquente, à rapprocher de celle qui se manifesterait par une analyse défectueuse sur les plans logique ou empirique ; et c'est cette dernière qui pose problème, ainsi que L. Campbell ou J.-P. Demoule l'explicitent à propos de M. Ruhlen ou de C. Renfrew.
Quant à l'originalité, elle est relative. Sans doute quasi nulle pour les pairs, qui apprécieront seulement la modalité de présentation et les choix contextualisés des éditeurs, elle est néanmoins importante pour un public non spécialisé, pour qui le quart de siècle passé depuis l'émergence de ces thèmes n'est pas significatif.

En ce qui concerne les recherches en modélisation, on constate que les travaux de ce type sont généralement publiés non pas dans des revues concernant les langues ou le langage, mais dans des revues dédiées aux sciences expérimentales, aux sciences de la vie, ou dans tout autre support réputé diffuser les éphémérides de la recherche «de pointe » (Nature, etc.). Tendanciellement, les textes sont cosignés, manifestant ainsi la réalité sinon d'une réflexion collective, du moins d'un travail partagé en équipe et de la conjonction disciplinaire sur le thème. L'on se trouve donc, formellement, dans une configuration sociologique proche du standard de la production scientifique "de masse», effectuée par des chercheurs qui travaillent «en laboratoire » sur des projets collectifs institutionnellement financés et qui sont tenus à une condition de productivité déterminante pour la qualité de leur avenir. Les deux chapitres insérés dans cet ouvrage souscrivent à ces réquisits : les objectifs sont bien de développer des procédures de description et des modélisations concernant la représentation des apparentements généalogiques saisissables à travers des analyses formalisées, et, le cas échéant, de serrer au plus près les phénomènes en appréhendant les dynamiques transversales telles celles de l'emprunt. Dès lors, les questions posées portent autant, et peut-être davantage, sur les propriétés formelles et la valeur heuristique supposée de ces modèles pris en eux-mêmes, que sur leur pertinence et leurs limites dans la description des phénomènes de l'évolution des langues. S'il y a là un comportement limite, il ne conduit pas à une dérive des hypothèses sur l'origine des langues telle que M. Ruhlen en fournit l'exemple, mais à une hypertrophie vers la construction de modèles pris pour objets de la recherche en lieu et place des réalités empiriques qui sont censées justifier leur emploi : on cherche (risque de chercher) davantage à affirmer les potentialités classificatoires du modèle qu'à discuter sa pertinence théorique pour l'utilisation que l'on en fait, et la critique des pratiques de la présentation et de la construction des données n'est pas envisagée.

$C^{\prime}$ est donc vers une réflexion épistémologique que cela nous entraîne : quelle que soit la qualité scientifique des propositions avancées dans leurs champs disciplinaires respectifs - des plus traditionnels ouverts au XIX ${ }^{\mathrm{e}}$ siècle avec le comparatisme linguistique, aux plus actuels avec les approches cognitives -, ce qui est censé «faire sens » dans un tel ouvrage n'est pas l'avancée disciplinaire, mais la pluridisciplinarité et, au-delà, l'interdisciplinarité autour de quelques propositions générales non réfutables à base empirique (origine des langues, 
diffusion indo-européenne, émergence du langage) ou à vocation «explicative » (représentations généalogiques arborescentes, évolutionnisme darwinien).

\section{Intérêt épistémologique}

On trouve ainsi, de-ci de-là dans les textes, des propositions à valeur épistémologique (méthodologique à tout le moins) qui demanderaient débat.

L. Cavalli-Sforza note la différence entre les sciences historiques, qui traitent d'événements uniques, et les sciences expérimentales, fondées sur une méthodologie de la preuve impliquant la répétition, et il conclut à la nécessité d'une approche croisée de l'ensemble des sciences ayant à voir avec une reconstruction de l'histoire : archéologie, paléoanthropologie, démographie, génétique, linguistique, anthropologie culturelle. Il exécute alors un grand écart en postulant que l'obtention d'un consensus interdisciplinaire sur un phénomène historique permet d'atteindre un degré de validation proche de celui qui peut être obtenu, pour un phénomène étudié en sciences expérimentales, par une confirmation expérimentale et par la maîtrise des conditions de production.

D. Parisi et al. introduisent leur contribution par un préambule sur l'intérêt et la valeur des simulations informatiques en tant que nouvelles façons d'exprimer des théories scientifiques.

On trouve aussi des modalités plus classiques d'analyse, telle l'expérience de pensée utilisée comme outil de recherche conjecturale. Il est probable que cette modalité d'analyse est utile pour prendre de la distance par rapport aux phénomènes, ainsi que le fait élégamment A. Castairs-McCarthy, et qu'elle a une fonction heuristique. L'expérience de pensée est efficace pour toutes les configurations de recherche où ce qui est en jeu est soit sans lien avec une description de faits empiriques, comme lorsqu'il s'agit de soutenir un raisonnement philosophique, soit en rapport étroit avec un état du monde dont il s'agit de rendre compte, mais tel qu'il corresponde à un comportement prévisible le plus souvent cerné dans un protocole d'expérience dont les variables sont définies pour un cadre théorique donné. Dans ce cas, elle prend sa valeur parce qu'elle introduit la possibilité de l'écart : la non-conformité éventuelle entre le comportement constaté et le résultat «modélisé » par l'expérience de pensée est un phénomène à expliquer. Elle a ainsi pour fonction de montrer la nécessité et/ou la « raisonnabilité » de l'hypothèse dans les conditions de l'expérience supposée (le terme « raisonnabilité » que j'introduis ici étalonne ce que l'on construit à ce que l'on croit qu'il est raisonnable de tenir pour acceptable sur une base essentiellement discursive). Mais, dans les cas proposés par l'auteur, sommes-nous dans cette configuration-là ? Pour synthétiser, dans le cadre d'une expérience de pensée, je ne fais jamais que reproduire et faire jouer sur fond de « raisonnabilité » ce que je juge bon d'avoir posé au départ. Sans plus.

C'est sans doute là que l'on rejoint les initiales questions sociologiques... Car il n'y a pas que des résultats de recherches désincarnés (réfutables ou non), il y a aussi les chercheurs qui les développent dans le présent, des instances qui déterminent leurs activités. Et l'on trouvera liées, d'une part, des propositions d'ouverture focalisées sur des phénomènes et, d'autre part, des postures prises par les acteurs de la recherche.

Au-delà du fait qu'en général, elles avalisent ce qui les introduit, des «propositions d'ouverture " ne sont pas réfutables : ce peut être des propositions intéressantes, enthousiasmantes ou qualifiées de l'adjectif que l'on voudra ; quant aux postures, elles manifestent simplement la place des acteurs dans la dynamique qu'ils développent (qui se développe), le jeu de la posture ayant - semble-t-il - une importance d'autant plus grande que les potentialités de vérification de la valeur scientifique des hypothèses émises est faible. Ici, l'exemple limite est sans doute celui offert par M. Ruhlen, dont l'approche est critiquée par L. Campbell et stigmatisée par B. Laks (et bien d'autres évidemment).

Dès lors, on conçoit l'importance de ce que j'appelle le «bouclage médiatique » dans la présentation des "résultats ». En effet, dans les milieux qui ont suivi son développement, la reprise d'un questionnement sur l'origine des langues et du langage a connoté de façon exemplaire une pratique particulière : l'interaction volontariste des scientifiques et des vulgarisateurs sur une question sensible pour le "grand public » parce qu'en arrière-plan d'une problématique scientifique éventuelle, il y a la présence latente d'une mythologie. Autrement dit, dès que les «Scientifiques » ont créé cet événement qu'auront été les propositions renouvelées sur ce thème, la potentialité d'une articulation "recherche-médiation-vulgarisation » a pu se mettre à fonctionner. Toutefois, par «mythologie », je n'entends pas un retour au mythe connu, mais la construction d'une mythologie moderne au sens de Barthes (1957), qui notait:

«Le mythe ne nie pas les choses, sa fonction est au contraire d'en parler ; simplement, il les purifie, les innocente, les fonde en nature et en éternité, il leur donne une clarté qui n'est pas celle de l'explication, mais celle du constat [...]. [...] il abolit la complexité des actes humains, leur donne la simplicité des essences, [...] il organise un monde sans contradictions parce que sans profondeur, un monde étalé dans l'évidence, il fonde une clarté heureuse ; les choses ont l'air de signifier toutes seules. »

Comment, dans les parties où j'ai quelques compétences, ne pas voir ces stratégies qui manipulent une "évidence », instrumentalisée au premier degré dans les pratiques d'un Ruhlen, et au deuxième degré par les pratiques de tous ceux d'entre nous qui sont concernés par la 
recherche et son management? L'élaboration d'une telle mythologie a ainsi vocation à se démultiplier en englobant et en déterminant tout aussi bien d'autres foyers mythologiques : la «Science » et ce qui est donné comme son parangon, «l'Excellence».

In fine, à l'issue de cette lecture - car c'est bien d'une «lecture » qu'il s'agit -, pour la qualité de son contenu, pour la distance qu'il a réussi à maintenir dans l'articulation des différents points de vue et, aussi, parce qu'il est sans doute un exemple de positionnement stratégique au sein d'une problématique éminemment discutable et discutée, je ne peux que recommander la lecture de cet ouvrage aussi bien aux étudiants et aux universitaires qu'à ceux qui souhaitent à la fois avoir un peu d'information de première main et prendre un peu de distance par rapport à ce qui est dit.

\section{Références}

Barthes, R., 1957. Mythologies, Paris, Le Seuil.

Bellwood, P., Renfrew, C. (Eds), 2002. Examining the Farming/Language Dispersal Hypothesis, Cambridge (UK), McDonald Institute for Archaeological Research, University of Cambridge.

Chomsky, N., 2001. Beyond explanatory adequacy, MIT Occasional Papers in Linguistics, 20, 1-28.
Fauconnier, G., Turner, M., 2002. The Way We Think: Conceptual Blending and the Mind's Hidden Complexities, New York, Basic Books.

Greenberg, J., 1963. The Languages of Africa, Bloomington, Indiana University.

Greenberg, J., 1987. Language in the Americas, Stanford, Stanford University Press.

Métoz, L., 2006. La convergence pluridisciplinaire dans la recherche de l'origine des langues : l'exemple américain de la "Nouvelle Synthèse ". Aspects historiques, théoriques et étude critique, Marges linguistiques, 11, 281-296 (http://www.revue-texto.net/1996-2007/ Archives/Archives.html [Marges linguistiques]).

Nicolaï, R., 2000. La Traversée de l'empirique, Paris, Ophrys.

Nicolaï, R., 2006. Origine du langage et origine des langues : réflexions sur la permanence et le renouvellement d'un questionnement des Lumières, Marges linguistiques, 11, 93-129 (http://www.revue-texto.net/1996-2007/ Archives/Archives.html [Marges linguistiques]).

Renfrew, C., 1999. Time depth, convergence theory, and innovation in Proto-Indo-European, 'Old Europe' as a PIE linguistic area, Journal of Indo-European Studies, 27, 3-4, 257-293.

Ruhlen, M., 1994. The Origin of Language: Tracing the Evolution of the Mother Tongue, New York, John Wiley \& Sons. Trad. fr. : L'Origine des langues, Paris, Belin, 1997.

Troubetzkoy, N.-S., 1939. Gedanken über das Indo-germanenproblem, Acta Linguistica, I, 81-89.

Williams, G.-C., 1992. Natural Selection: Domains, Levels, and Challenges, New York, Oxford University Press. 